\title{
Saccade dysmetria indicates attenuated visual exploration in autism spectrum disorder
}

\author{
Nico Bast, ${ }^{1}{ }_{1}$ Luke Mason, ${ }^{2}$ Christine M. Freitag, ${ }^{1}$ Tim Smith, ${ }^{2}$ Ana Maria Portugal, ${ }^{2}$ \\ Luise Poustka, ${ }^{3}$ Tobias Banaschewski, ${ }^{4, *}$ Mark Johnson, ${ }^{2,5, *}$ and The EU-AIMS LEAP Group \\ ${ }^{1}$ Department of Child and Adolescent Psychiatry, Psychosomatics and Psychotherapy, University Hospital,Goethe \\ University Frankfurt am Main, Frankfurt, Germany; ${ }^{2}$ Center for Brain and Cognitive Development, Birkbeck College, \\ University of London, London, UK; ${ }^{3}$ Department of Child and Adolescent Psychiatry/Psychotherapy, University \\ Medical Center Göttingen,Medical University of Göttingen, Göttingen, Germany; ${ }^{4}$ Department of Child and \\ Adolescent Psychiatry and Psychotherapy, Central Institute of Mental Health, Heidelberg University, Heidelberg, \\ Germany; ${ }^{5}$ Department of Psychology, University of Cambridge, Cambridge, UK
}

\begin{abstract}
Background: Visual exploration in autism spectrum disorder (ASD) is characterized by attenuated social attention. The underlying oculomotor function during visual exploration is understudied, whereas oculomotor function during restricted viewing suggested saccade dysmetria in ASD by altered pontocerebellar motor modulation. Methods: Oculomotor function was recorded using remote eye tracking in 142 ASD participants and 142 matched neurotypical controls during free viewing of naturalistic videos with and without human content. The sample was heterogenous concerning age (6-30 years), cognitive ability (60-140 IQ), and male/female ratio (3:1). Oculomotor function was defined as saccade, fixation, and pupil-dilation features that were compared between groups in linear mixed models. Oculomotor function was investigated as ASD classifier and features were correlated with clinical measures. Results: We observed decreased saccade duration $(\Delta M=-0.50$, CI $[-0.21,-0.78])$ and amplitude $(\Delta M=-0.42$, CI $[-0.12,-0.72])$, which was independent of human video content. We observed null findings concerning fixation and pupil-dilation features $($ POWER $=.81)$. Oculomotor function is a valid ASD classifier comparable to social attention concerning discriminative power. Within ASD, saccade features correlated with measures of restricted and repetitive behavior. Conclusions: We conclude saccade dysmetria as ASD oculomotor phenotype relevant to visual exploration. Decreased saccade amplitude and duration indicate spatially clustered fixations that attenuate visual exploration and emphasize endogenous over exogenous attention. We propose altered pontocerebellar motor modulation as underlying mechanism that contributes to atypical (oculo-)motor coordination and attention function in ASD. Keywords: Eye tracking; pupillometry; visual attention; biomarker; brainstem; cerebellum; locus coeruleus.
\end{abstract}

\section{Introduction}

Visual exploration describes free viewing of stimuli and characterizes how we distribute our visual attention in complex environments (Itti \& Koch, 2001). Oculomotor function is the underlying eye muscle movement that motorically bounds visual exploration (Corbetta, 1998). In autism spectrum disorder (ASD), visual exploration is characterized by attenuated attention to social stimuli (Frazier et al., 2017), which has been established as predictor of ASD diagnosis (Jones et al., 2016). This different visual exploration in ASD has been explained by a theory of 'low social motivation' (Chevallier, Kohls, Troiani, Brodkin, \& Schultz, 2012), although empirical evidence is inconsistent (Bottini, 2018). Alternatively, different visual exploration in ASD might be determined by aberrant oculomotor function (Johnson, Lum, Rinehart, \& Fielding, 2016).

In ASD, it is largely unknown whether oculomotor function is different during visual exploration because oculomotor function has been predominantly investigated during restricted viewing, which

*Shared last authorship.

Conflict of interest statement: See Acknowledgements for full disclosures. refers to experimentally manipulated gaze behavior. A meta-analysis on restricted viewing described that ASD participants were less able to inhibit (pro-) saccades in the antisaccade task $(\mathrm{SMD}=1.71$; Johnson et al., 2016), showed increased variation in saccade accuracy during visually guided (pro-) saccades $(\mathrm{SMD}=0.88)$, and decreased saccade-totarget accuracy during smooth pursuit $(\mathrm{SMD}=1.35-1.87)$. These findings were interpreted as saccade dysmetria, that is, ASD individuals are less able to adapt their saccades to visual targets (Mosconi et al., 2013). However, restricted-viewing paradigms evoke oculomotor function that is uncommon (e.g., antisaccades, smooth pursuit) during visual exploration, whereas visual exploration does not provide unambiguous visual targets to quantify impaired accuracy. Thus, restricted-viewing findings might not be translatable to naturalistic visual exploration.

Oculomotor function during visual exploration has been investigated in limited ASD samples. Infants at risk for ASD showed shorter fixation duration (Wass et al., 2015) and increased reorienting to previously fixated areas (Gliga, Smith, Likely, Charman, \& Johnson, 2015) on static images of faces and objects. ASD adults with average IQ showed less fixations per second, longer saccade duration, and 
attenuated saccade velocity on naturalistic static images (Wang et al., 2015). Those oculomotor-function studies in ASD suggested attenuated visual exploration of static images, which is supported by gaze pattern analysis and has been associated with restricted and repetitive behaviors (RRBs; Manyakov et al., 2018). However, these findings may not generalize to the ASD population as contributing effects of cognitive ability and age are unexplored.

In addition, those studies did not conclusively replicate saccade dysmetria, which might be due to the application of static stimuli. In a seminal paper (Chevallier et al., 2015), attenuated social attention only occurred in ASD children/adolescents during naturalistic videos of interacting children compared to static images and face-versus-object videos. In a corresponding meta-analysis, attenuated social attention in ASD was emphasized by stimuli that resembled human social situations (Frazier et al., 2017). In contrast, ASD oculomotor function - as motor boundary of visual exploration - would be independent of human content. Thus, ASD oculomotor function that translates to naturalistic visual exploration needs to be explored during naturalistic videos with and without human content.

Oculomotor function can further inform about underlying neuronal networks (see Figure 1). Saccade production has been attributed to the frontoparietal attention networks including temporoparietal junction (TPJ; Corbetta et al., 1998). These cortical attention networks output attention-target information, which is converted to a topographic map within the superior colliculi, and executed as saccade via pontine and brainstem oculomotor neurons (PBON; Johnson et al., 2016).
Fixation production has been attributed to a corticopontocerebellar network (Munoz \& Everling, 2004) that also includes PBON and fastigial oculomotor nucleus (FON) both related to saccade inhibition execution. This pontocerebellar network sets corresponding peak velocity for a saccade to a cortically defined visual target (Nakamagoe, Iwamoto, \& Yoshida, 2000) and has been associated with rapid transitions in ASD motor execution (Blakemore, Cook, \& Press, 2013). A disproportionate setting of peak velocity led to an over- or undershooting of saccades (Guerrasio, Quinet, Büttner, \& Goffart, 2010; Johnson et al., 2012) and could be observed during visual exploration as altered saccade duration or amplitude. In ASD, altered pontocerebellar motor modulation has been proposed to explain decreased saccade duration and amplitude during restricted viewing (Mosconi et al., 2013; Takarae, Minshew, Luna, \& Sweeney, 2004).

Altered pontocerebellar motor modulation could further contribute to behavioral findings of attenuated visual exploration (Wass \& Smith, 2014) and delayed peripheral orienting (Landry \& Parker, 2013). A decreased saccade duration and amplitude bound visual exploration to spatially clustered fixations (Gliga, Bedford, et al., 2015; Wang \& Munoz, 2015). This could relate saccade dysmetria in ASD to attenuated visual exploration and propose pontocerebellar motor modulation as shared underlying mechanism.

The present study investigates ASD-specific oculomotor function during free viewing of naturalistic videos in comparison with matched neurotypical controls. We assess a large and heterogenous sample to identify findings generalizable to the ASD

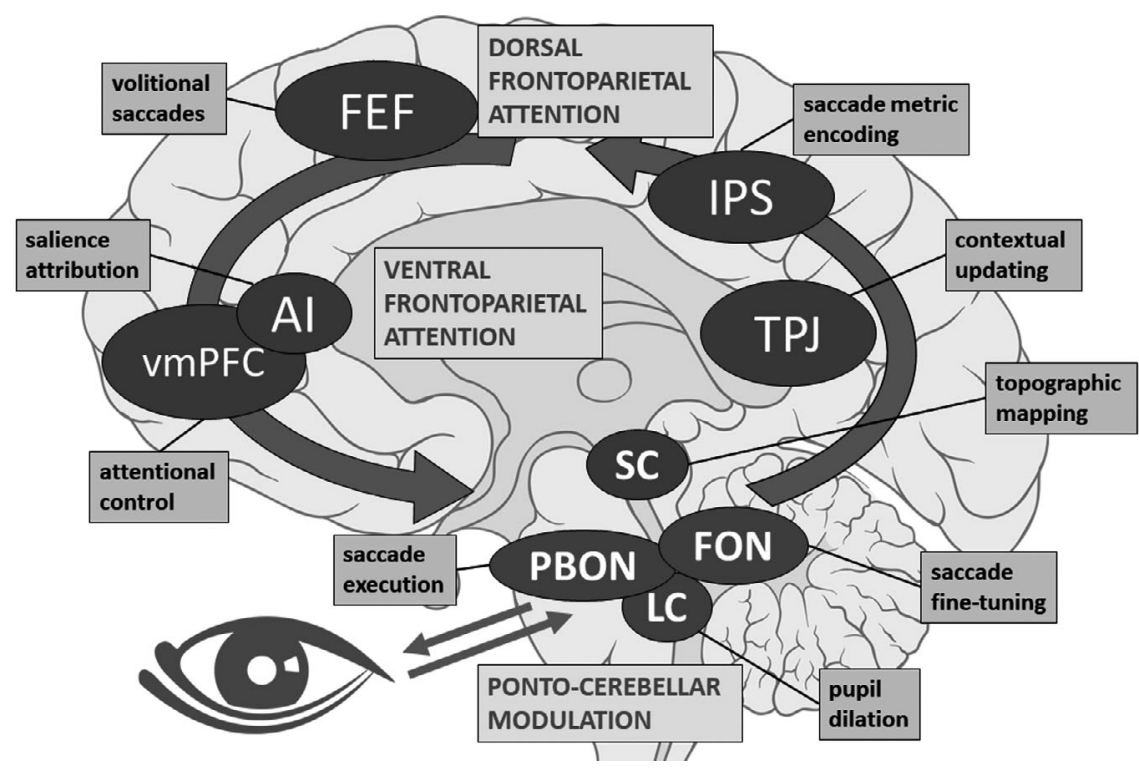

Figure 1 Neural networks underlying oculomotor function. Boxes describe associated function of neural network nodes (oval) relevant to oculomotor function. Abbreviations: Al, anterior insula; FEF, frontal eye fields; FON, fastigial oculomotor neurons; IPS, intraparietal sulcus; LC, locus coeruleus; PBON, pontine and brainstem oculomotor neurons; SC, superior colliculi; TPJ, temporoparietal junction; vmPFC, ventromedial prefrontal cortex 
population. We apply naturalistic videos with and without human content, as oculomotor function should be independent of stimulus content. ASDspecific oculomotor function is operationalized as group differences in saccade, fixation, or pupildilation (PD) features. Features of oculomotor function that are less frequent during free viewing (e.g., smooth pursuit) are not investigated. We expect ASD-specific oculomotor function that relates to previous restricted-viewing findings of saccade dysmetria. Thus, we hypothesize decreased saccade duration and amplitude during visual exploration. Previous literature also indicated fewer and shorter fixations to social stimuli, which we do not expect in ASD-specific oculomotor function independent of stimulus content. Further, we exploratively investigate PD during visual exploration, as an altered pupillary light reflex has recently been outlined as predictor of ASD symptoms (Nyström et al., 2018). We assume oculomotor function to be an informative ASD classifier that correlates with ASD clinical screening and symptom measures.

\section{Methods and materials Sample}

The final study sample (ASD: $n=142$, typical development [TD]: $n=142$ ) was part of the EU-AIMS LEAP study (see Figure 2; Charman et al., 2017). In contrast to the original study, we additionally excluded ASD participants that scored below clinical cutoff (Gotham, Pickles, \& Lord, 2009; Autism Diagnostic Observation Schedule [ADOS-2] Calibrated Severity Score $<4, n=105$ ) and participants with substantially deviating IQ scores (IQ <60|IQ> 140; ASD: $n=5$; TD: $n=6$ ). Remaining participants with sufficient data quality (see data preprocessing; ASD: $n=194$, TD: $n=217$ ) were matched within age subgroups for full scale IQ, age, gender distribution, and eye-trackers' sampling rate (see procedure) by nearest neighbor method with $0.1 S D$ tolerance. Matching excluded 28 children (ASD: $n=9$, TD: $n=19$ ), 53 adolescents (ASD: $n=31$, TD: $n=22$ ), and 46 adults (ASD: $n=12$, TD: $n=34$ ). Matched groups were comparable concerning preand postprocessing data quality, but differed on the inattentiveness subscale of the Attention Deficit Hyperactivity Disorder (ADHD) rating scale (Kessler et al., 2005), which is unsurprising given elevated ADHD co-occurrence in ASD (Simonoff et al., 2008). All sample descriptive variables (see Table 1) were considered in the statistical analysis. Clinical characterization suggested ASD symptom heterogeneity (see Table 2).

\section{Procedure}

The full EU-AIMS LEAP assessment protocol has been outlined elsewhere (Loth et al., 2017). All participants and their caregiver provided written informed consent. Ethical approval for this study was obtained through ethics committees at each site (see Table S1). Assessments were done at six site-specific eye-tracking laboratories with luminance that is adapted for optimal eye detection (Lux: $m=164, S D=109$ ). Oculomotor function was recorded by remote eye-trackers (Tobii T120 or TX300, Stockholm, Sweden) with a target distance of $65 \mathrm{~cm}$, while heads could be moved freely. Nine naturalistic videos were presented without a specific task (see Table S2). Videos were presented in pseudo-random order on

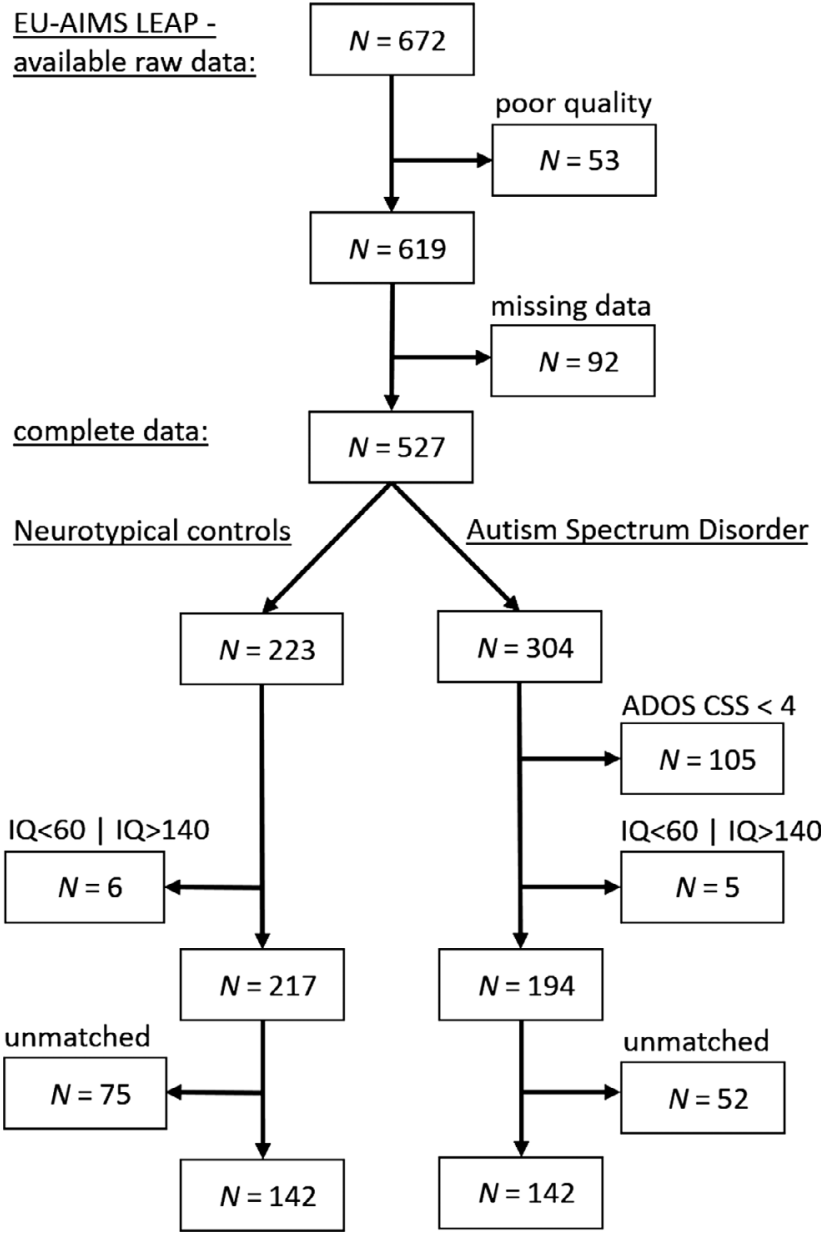

Figure 2 Flow Chart: Study Sample. Top part: Data reduction described in 'data preprocessing' section. Bottom part: Sample matching described in 'sample' section

17- or 23-inch displays with a fixed display area of $345 \times 259 \mathrm{~mm}$. Videos were of two content conditions (4 human, 5 nonhuman), varied in duration (10 s-124 s), and were displayed with audio, although no speech was involved that is, human interactions were either nonverbal or speech was superimposed by ambient sounds - to ensure comparability across multinational sites.

Remote eye-trackers limited sampling rates compared to restricted-viewing studies that usually require head restraints to reduce noise (Schmitt, Cook, Sweeney, \& Mosconi, 2014). This was not feasible in our child/adolescent samples and would have contrasted the naturalistic visual exploration rationale. Thus, our lower temporal resolution introduced larger temporal measurement errors. However, simulations demonstrated that this is compensated by our large number of saccade measurements per participant $(120 \mathrm{~Hz}: m=445$, $S D=249 ; 300 \mathrm{~Hz}: m=594, S D=282)$, which did not differ between groups $(t s<1)$ and is well above suggested cutoffs (Andersson, Nyström, \& Holmqvist, 2010).

\section{Data preprocessing}

We assessed eye-tracking datasets of 672 individuals (see Figure 2). Raw-data quality was estimated on four quality dimensions (Flicker, Precision, Accuracy, and Binocular Disparity), which were derived from principal component analysis on quality related metrics (Radach, Deubel, Vorstius, \& Hofmann, 2017; see Table S3). Based on these four quality dimensions, raw datasets were clustered in four preprocessing 
Table 1 Sample descriptives

\begin{tabular}{|c|c|c|c|c|c|c|c|}
\hline \multirow{2}{*}{$\begin{array}{l}\text { Variable (unit) } \\
\text { Age (years) }\end{array}$} & \multicolumn{3}{|c|}{ Autism spectrum disorder (ASD) } & \multicolumn{3}{|c|}{ Typical development (TD) } & \multirow[t]{2}{*}{$\begin{array}{l}\text { Test } \\
p \text {-Value }\end{array}$} \\
\hline & $N$ & Mean/SD & Range & $N$ & Mean/SD & Range & \\
\hline All & 142 & $16.23 / 5.77$ & $6-30$ & 142 & $16.21 / 5.62$ & $6-30$ & .984 \\
\hline Children & 39 & $9.6 / 1.57$ & $6-11$ & 38 & $9.69 / 1.59$ & $6-11$ & .797 \\
\hline Adolescent & 55 & $15.24 / 1.71$ & $12-17$ & 61 & $15.4 / 1.64$ & $12-17$ & .615 \\
\hline Adult & 48 & $22.74 / 3.7$ & $18-30$ & 43 & $23.13 / 3.42$ & $18-30$ & .603 \\
\hline All & 142 & $102.17 / 15.59$ & $61-139$ & 142 & $102.58 / 15.31$ & $61-134$ & .825 \\
\hline Children & 39 & $107.57 / 14.04$ & $80-139$ & 38 & $107.71 / 10.92$ & $85-130$ & .961 \\
\hline Adolescent & 55 & $98.09 / 18.17$ & $61-139$ & 61 & $98.67 / 16.45$ & $61-134$ & .857 \\
\hline Adult & 48 & $102.47 / 12.13$ & $77-124$ & 43 & $103.59 / 15.76$ & $62-132$ & .707 \\
\hline Sex (category) & Female & Male & & Female & Male & & \\
\hline All & $37(26 \%)$ & 105 (74\%) & & $41(29 \%)$ & $101(71 \%)$ & & .69 \\
\hline Children & $13(33 \%)$ & $26(67 \%)$ & & $11(29 \%)$ & $27(71 \%)$ & & .866 \\
\hline Adolescent & $8(15 \%)$ & 47 (85\%) & & $19(31 \%)$ & 42 (69\%) & & .058 \\
\hline ADHD inatt. (total) & $N$ & Mean/SD & Range & $N$ & Mean/SD & Range & \\
\hline All & 142 & $4.47 / 3.16$ & $0-9$ & 142 & $1.13 / 1.84$ & $0-9$ & .000 \\
\hline Children & 39 & $5.23 / 3.05$ & $0-9$ & 38 & $0.63 / 1.28$ & $0-7$ & .000 \\
\hline Adolescent & 55 & $4.38 / 3.05$ & $0-9$ & 61 & $1.46 / 2.17$ & $0-9$ & .000 \\
\hline Adult & 48 & $3.96 / 3.31$ & $0-9$ & 43 & $1.09 / 1.67$ & $0-6$ & .000 \\
\hline Sampling rate $(\mathrm{Hz})$ & 300 & 120 & & 300 & 120 & & \\
\hline All & $35(25 \%)$ & 107 (75\%) & & $34(24 \%)$ & $108(76 \%)$ & & 1 \\
\hline Children & $7(18 \%)$ & $32(82 \%)$ & & $8(21 \%)$ & $30(79 \%)$ & & .955 \\
\hline Adolescent & $13(24 \%)$ & $42(76 \%)$ & & $14(23 \%)$ & 47 (77\%) & & 1 \\
\hline Adult & $15(31 \%)$ & $33(69 \%)$ & & $12(28 \%)$ & $31(72 \%)$ & & .906 \\
\hline Preprocessing (clusters) & Good & Medium & Flicker & Good & Medium & Flicker & \\
\hline All & $52(37 \%)$ & $49(35 \%)$ & 41 (29\%) & $71(50 \%)$ & $40(28 \%)$ & $31(22 \%)$ & .073 \\
\hline Children & $11(28 \%)$ & $12(31 \%)$ & $16(41 \%)$ & $15(39 \%)$ & $10(26 \%)$ & $13(34 \%)$ & .578 \\
\hline Adolescent & $19(35 \%)$ & 24 (44\%) & $12(22 \%)$ & $32(52 \%)$ & $20(33 \%)$ & $9(15 \%)$ & .149 \\
\hline Adult & $22(46 \%)$ & $13(27 \%)$ & $13(27 \%)$ & $24(56 \%)$ & $10(23 \%)$ & $9(21 \%)$ & .627 \\
\hline Children & 39 & $99.51 / 0.5$ & $97.8-100$ & 38 & $99.56 / 0.55$ & $97-100$ & .689 \\
\hline Adolescent & 55 & $99.77 / 0.29$ & $98.8-100$ & 61 & $99.71 / 0.39$ & $98.4-100$ & .361 \\
\hline Adult & 48 & $99.71 / 0.41$ & $98-100$ & 43 & $99.7 / 0.35$ & $98.7-100$ & .883 \\
\hline
\end{tabular}

Test = group comparison with $t$-test or Chi-square test, ADHD inatt. = ADHD rating scale - inattentiveness sub-scale. preprocessing $=$ preprocessing data quality clusters (flicker $=$ missing segments but otherwise good raw data), postprocessing $=$ postprocessing data quality.

data quality clusters (cluster label [ASD/TD]: Good [125/126], Medium [136/78], Flicker [108/59], Insufficient [29/11]). We dropped the 'insufficient' cluster from further analysis $(n=40)$. A total of 632 raw datasets were preprocessed by GraFIX software (de Urabain, Johnson, \& Smith, 2014), which estimated fixations and saccades by a velocity-based identification algorithm (see Table S3). PD was provided by the raw data. We created a postprocessing data quality variable (see Table 1) to check whether preprocessing delivered sufficient data quality, which was defined as percentage of successful fixation/ saccade identification per video. Five participants $(n=5)$ with deviating postprocessing data quality - less than $97 \%$ - were excluded and additional participants with a sampling rate smaller than $120 \mathrm{~Hz}(n=8)$. Additional datasets were excluded as demographic data was incomplete $(n=92)$.

\section{Oculomotor features}

Seven oculomotor features (OFs) were selected based on previous literature and calculated in the preprocessed data. This includes fixation duration (Wass \& Smith, 2014), fixations per second (Auyeung et al., 2015), relative pupil dilation (Mill, O'Connor, \& Dobbins, 2016), that is, absolute pupil dilation corrected for per-participant mean, saccade duration (Schmitt et al., 2014), saccade amplitude (Mosconi et al., 2013; Wass et al., 2015), and peak saccade velocity (Schmitt et al., 2014; Takarae, Minshew, Luna, \& Sweeney, 2004). We further included velocity main sequence as coefficient of peak saccade velocity and saccade amplitude (Bahill, Clark, \& Stark, 1975), which allows to interpret peak saccade velocity independent of varying saccade amplitude. For each of the $7 \mathrm{OFs}$, we calculated a mean and $S D$ per participant and video (see Table 3), which were applied as dependent variables in the statistical analysis. We were not able to estimate accuracy and latency measures (Johnson et al., 2016), as the visual exploration precludes an a priori definition of visual targets.

\section{Statistical analysis}

Data were analyzed by R 3.4.3. software (R Core Team, 2014) with additional packages (Bates, Maechler, Bolker, \& Walker, 2014; Ho, Imai, King, Stuart, \& Whitworth, 2018; Kuznetsova, Brockhoff, \& Christensen, 2017; Robin et al., 2011; Russell, 2018). Oculomotor features (OFs) were scaled and separately compared in $i=14$ linear mixed models with restricted maximum likelihood estimation and group as fixed effect: 
Table 2 Clinical characterization of the autism spectrum disorder sample

\begin{tabular}{|c|c|c|c|c|c|c|}
\hline \multirow[b]{2}{*}{ Variable (unit) } & \multicolumn{2}{|l|}{ Child } & \multicolumn{2}{|l|}{ Adolescent } & \multicolumn{2}{|l|}{ Adult } \\
\hline & Mean/SD & Range & Mean/SD & Range & Mean/SD & Range \\
\hline \multicolumn{7}{|l|}{ ADOS-2 (CSS) } \\
\hline Total & $6.74 / 1.9$ & $4-10$ & $7 / 2.07$ & $4-10$ & $6.69 / 1.95$ & $4-10$ \\
\hline Social affect & $7.13 / 1.81$ & $3-10$ & $7.71 / 1.67$ & $4-10$ & $7.31 / 1.64$ & $4-10$ \\
\hline Restricted/repetitive & $5.77 / 3.06$ & $1-10$ & $4.73 / 2.82$ & $1-10$ & $5.62 / 2.39$ & $1-10$ \\
\hline \multicolumn{7}{|l|}{ ADI-R (total) } \\
\hline Social & $15.15 / 7.23$ & $1-26$ & $18.59 / 6.55$ & $2-29$ & $15.93 / 7.53$ & $0-28$ \\
\hline Verbal Communication & $14.82 / 5.25$ & $5-25$ & $14.61 / 5.4$ & $3-26$ & $12.32 / 6.02$ & $0-24$ \\
\hline Restricted/repetitive & $4.79 / 3.15$ & $0-12$ & $4.65 / 2.7$ & $0-12$ & $4.83 / 2.89$ & $0-12$ \\
\hline \multicolumn{7}{|l|}{ Questionnaires (total) } \\
\hline SRS parent & $105.24 / 27.7$ & $51-163$ & $96.02 / 25.14$ & $42-153$ & $86.66 / 34.43$ & $21-154$ \\
\hline SRS self & - & - & $70.07 / 26.65$ & $26-125$ & $86.35 / 30.99$ & $12-151$ \\
\hline RBS-R & $21.25 / 13.77$ & $1-62$ & $17.48 / 14.23$ & $0-73$ & $13.29 / 12.89$ & $0-53$ \\
\hline SSP & $127.66 / 22.87$ & $82-171$ & $135.97 / 25.44$ & $88-178$ & $146.12 / 28.63$ & $93-177$ \\
\hline
\end{tabular}

ADI-R, Autism Diagnostic Interview - Revised; ADOS-2, Autism Diagnostic Observation Schedule; CSS, Calibrated Severity Score; RBS-R, Repetitive Behavior Scale - Revised; SRS parent, Social Responsiveness Scale Total Score - parent rating; SRS self, Social Responsiveness Scale Total Score - self rating; SSP, Short Sensory Profile.

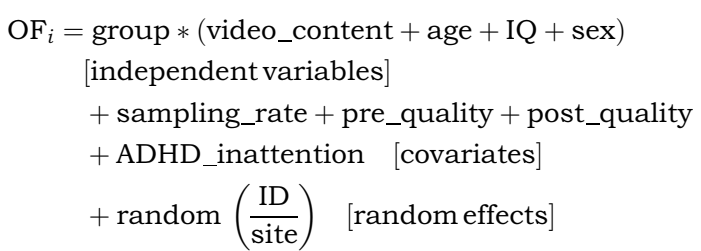

We included video content (human, nonhuman) as withinsubject fixed effect and modeled an interaction with group. Main group effects without video content interactions would indicate ASD-specific oculomotor function that is independent of human content. We modeled age (child: 6-11 years, adolescent: $12-17$ years, adult: $18-30$ years), IQ (below average: $<85$, average: $85-115$, above average: $>115$ ), and sex (male, female) as categorical, between-subject fixed effects and allowed twoway interactions with group. Categorization of age and IQ does allow to interpret interactions with group as oculomotor function specific to ASD covariate subgroups. Sampling rate, preprocessing data quality, and postprocessing data quality were controlled for by inclusion as covariates. We further included the inattentiveness subscale of the ADHD rating scale as covariate, as we expected ADHD inattentiveness symptoms to affect visual exploration. Participant ID nested in study site were included as random effects. Fixed effects were tested for significance by ANOVA with Satterthwaite's method. Fixedeffect significance is corrected for false detection rate (FDR) by the number of estimated models $(i=14)$. We report pairwise comparisons of predicted marginal means $(\Delta M)$ to quantify group differences for significant effects, which are presented with $95 \%$ confidence intervals (CI).

Oculomotor function was defined as the composite of the 14 OFs. Discriminative power of oculomotor function was assessed by receiver operating characteristic (ROC) curves, which were estimated by a logistic regression with the 14 OFs as predictors of group affiliation in the overall sample and in age, IQ, and sex subgroups. Area under the curve (AUC) is reported with confidence intervals (Sun \& Xu, 2014).

In addition, OFs were correlated by Pearson's coefficient with ASD symptom and screening measures. These measures

Table 3 Descriptives of oculomotor features

\begin{tabular}{|c|c|c|c|c|c|c|c|c|c|c|}
\hline \multirow{2}{*}{$\begin{array}{l}\text { Oculomotor feature (unit) } \\
\text { Mean }\end{array}$} & \multirow[b]{2}{*}{ Plausible range } & \multicolumn{4}{|c|}{ Autism Spectrum Disorder (ASD) } & \multicolumn{4}{|c|}{ Typical development (TD) } & \multirow{2}{*}{$\begin{array}{l}\text { KW- } \\
\text { test } \\
p \text {-Value }\end{array}$} \\
\hline & & Mean & $S D$ & Min & $\operatorname{Max}$ & Mean & $S D$ & Min & $\operatorname{Max}$ & \\
\hline Fixation duration (ms) & $100-800$ & 339.65 & 36.54 & 250.86 & 473.93 & 335.52 & 38.57 & 188.83 & 459.20 & .414 \\
\hline Fixations per second $(1 / \mathrm{s})$ & $0-5$ & 1.37 & 0.40 & 0.32 & 2.23 & 1.48 & 0.42 & 0.34 & 2.32 & .051 \\
\hline Relative pupil dilation $(\% / 100)$ & $0.7-1.3$ & 1.01 & 0.04 & 0.89 & 1.19 & 1.01 & 0.05 & 0.87 & 1.14 & .941 \\
\hline Saccade duration (ms) & $10-100$ & 31.20 & 3.56 & 23.37 & 43.98 & 31.61 & 3.53 & 24.23 & 47.59 & .606 \\
\hline Saccade amplitude $\left({ }^{\circ}\right)$ & 0-20 & 5.52 & 0.70 & 3.18 & 7.06 & 5.72 & 0.75 & 3.66 & 7.89 & .066 \\
\hline Peak saccade velocity $(\% / s)$ & $0-1,500$ & 386.56 & 93.11 & 249.60 & 689.82 & 380.93 & 87.99 & 225.14 & 663.16 & .744 \\
\hline Velocity main sequence $(1 / \mathrm{s})$ & $0-200$ & 76.23 & 15.00 & 48.17 & 120.18 & 74.67 & 15.89 & 51.46 & 119.27 & .146 \\
\hline Variation $(S D)$ & & Mean & $S D$ & Min & $\operatorname{Max}$ & Mean & $S D$ & Min & $\operatorname{Max}$ & $p$-Value \\
\hline Fixation duration (ms & & 197.94 & 19.17 & 136.85 & 247.72 & 196.31 & 22.75 & 69.11 & 254.88 & .775 \\
\hline Fixations per second $(1 / \mathrm{s})$ & & 0.63 & 0.21 & 0.11 & 1.21 & 0.65 & 0.20 & 0.15 & 1.15 & .432 \\
\hline Relative pupil dilation (\%/100) & & 0.11 & 0.03 & 0.05 & 0.21 & 0.12 & 0.04 & 0.05 & 0.22 & .044 \\
\hline Saccade duration (ms) & & 18.48 & 3.24 & 10.53 & 29.19 & 18.48 & 3.23 & 12.04 & 27.70 & .986 \\
\hline Saccade amplitude $\left(^{\circ}\right)$ & & 4.74 & 0.44 & 3.00 & 5.67 & 4.79 & 0.40 & 3.68 & 5.83 & .503 \\
\hline Peak saccade velocity $(\% / \mathrm{s})$ & & 283.25 & 79.30 & 151.87 & 475.82 & 271.18 & 74.47 & 163.19 & 470.21 & .244 \\
\hline Velocity main sequence $(1 / \mathrm{s})$ & & 33.36 & 5.81 & 21.77 & 47.90 & 32.69 & 5.71 & 22.93 & 48.20 & .249 \\
\hline
\end{tabular}


are described above (Table 2) and further outlined elsewhere (Loth et al., 2017). We report significant coefficients after Bonferroni's multiple comparison correction.

\section{POWER analysis}

We estimated the achieved POWER for group differences by simulation with the observed random variance (Green \& MacLeod, 2016). POWER confidence intervals (CI) were based on 1,000 iterations. For small group differences $(\Delta M=0.2)$, we achieved a POWER (1- $\beta)$ of $81 \%$, CI [78.43, 83.39].

\section{Results}

Full models are provided in Tables S4-S6 with effects for age (Table S7) and video content (Table S8). These fixed effects were independent of ASD and thus not further discussed here. We further found no effect of IQ on oculomotor function.

\section{Oculomotor function in autism spectrum disorder}

During visual exploration of naturalistic videos, we found ASD-specific features with decreased means in saccade duration $(\Delta M=-0.50, \mathrm{CI}[-0.21,-0.78])$ and saccade amplitude $(\Delta M=-0.42$, CI $[-0.12,-0.72])$. There were no significant interactions, which indicated that ASD-specific oculomotor function was independent of video content, IQ, sex, or age. Decreased saccade duration and amplitude were further controlled for the effects of pre- and processing data quality, sampling rate, and ADHD inattentiveness ratings. In contrast, we did not observe group differences concerning fixation or pupil-dilation features.

\section{Discriminative power}

Oculomotor function as feature composite measure was able to classify group affiliation in the overall sample with AUC $=.670$, CI [0.608, 0.733]. Discriminative power was significantly increased (AUC $>$.733) when comparing classification in specific subgroups like children, adults, females, below average IQ, and above average IQ (see Table 4). In contrast, discriminative power was not increased when classification was restricted to human or nonhuman videos (see Figure 3).

\section{Correlations with clinical measures}

In correlation analysis, ADI-R restricted/repetitive domain score correlated positively with mean in velocity main sequence $\left(r(134)=.26, p_{\text {adj }}<.037\right)$, whereas SSP total score correlated negatively with variance in velocity main sequence $\left(r(84)=-.32, p_{\text {adj }}<.041\right)$. Descriptive correlations are presented in Table S9.

\section{Discussion}

We investigated ASD-specific oculomotor function during visual exploration of naturalistic videos. This design delivers increased ecological validity compared to restricted-viewing paradigms. We did not find group differences in fixation or pupil-dilation features, which is reliable regarding the achieved POWER. In contrast, we did find ASD-specific oculomotor function with decreased saccade duration and saccade amplitude. This saccade dysmetria was independent of human video content and thus cannot be explained by attenuated social attention. In our heterogenous sample, these findings were independent of age, cognitive ability, and sex. Thus, decreased saccade amplitude and duration during naturalistic visual exploration are concluded as an oculomotor phenotype relevant across the ASD population.

Decreased saccade amplitude and duration describe an ASD gaze behavior that is characterized by erratic saccades to peripheral targets (see Tables S5 and S6). Additional compensatory saccades will be required to reach peripheral targets (Takarae, Luna, Minshew, \& Sweeney, 2008), which has been described during restricted viewing as less precise saccades (Takarae, Minshew, Luna, Krisky, $\&$ Sweeney, 2004) and increased variation in accuracy (Mosconi et al., 2013). This interrelation of decreased saccade amplitude/duration and impaired saccade accuracy relates our visual exploration findings to previous restricted-viewing findings (Johnson et al., 2012).

Decreased saccade amplitude and duration further describe spatially clustered fixations during visual exploration. This is in line with ASD findings for static pictures with decreased gaze deviations from screen center (Wang et al., 2015) and increased reorienting to previously fixated areas (Gliga, Smith, et al., 2015). Accordingly, decreased saccade amplitude and duration reflect attenuated visual exploration. Specifically, ASD visual exploration could be characterized by reduced exogenous compared to endogenous attention (Corbetta, Patel, \& Shulman, 2008). Exogenous attention describes stimulus-driven and spatially distributed visual exploration, whereas endogenous attention describes task-focused and spatially clustered visual exploration (Chica, Bartolomeo, \& Lupiáñez, 2013). Reduced exogenous attention in ASD was reported for behavioral attention tasks (Renner, Grofer Klinger, \& Klinger, 2006). In infants at risk for ASD, a delayed response was reported to disengage gaze from a central visual target to orient to a peripheral one, which was a predictor of later ASD diagnosis (Bedford et al., 2017). Furthermore, a recent meta-analysis in ASD described delayed peripheral orienting in Posnertype attention tasks (Landry \& Parker, 2013). We conclude that these behavioral findings and decreased saccade amplitude/duration both describe attenuated visual exploration in ASD with reduced exogenous compared to endogenous attention. 
Table 4 Area under the Curve (AUC) of oculomotor features in subgroups

\begin{tabular}{|c|c|c|c|c|c|c|c|c|c|c|c|}
\hline & \multirow[b]{2}{*}{ All } & \multicolumn{3}{|c|}{ Age subgroups } & \multicolumn{3}{|c|}{ IQ subgroups } & \multicolumn{2}{|c|}{ Sex subgroups } & \multicolumn{2}{|c|}{$\begin{array}{l}\text { Video content } \\
\text { categories }\end{array}$} \\
\hline & & Adolescent & Adult & Children & $\begin{array}{l}\text { Above } \\
\text { average }\end{array}$ & $\begin{array}{l}\text { Below } \\
\text { average }\end{array}$ & Average & Female & Male & Human & Nonhuman \\
\hline AUC & 0.670 & 0.708 & 0.768 & 0.737 & 0.828 & 0.890 & 0.664 & 0.739 & 0.678 & 0.697 & 0.628 \\
\hline $\begin{array}{l}\text { Lower } \\
\text { bound }\end{array}$ & 0.608 & 0.615 & 0.671 & 0.619 & 0.719 & 0.776 & 0.587 & 0.630 & 0.605 & 0.627 & 0.564 \\
\hline $\begin{array}{l}\text { Upper } \\
\text { bound }\end{array}$ & 0.733 & 0.802 & 0.864 & 0.855 & 0.937 & 1.000 & 0.741 & 0.848 & 0.751 & 0.768 & 0.693 \\
\hline
\end{tabular}

Lower and upper bounds of AUC 95\% confidence intervals.
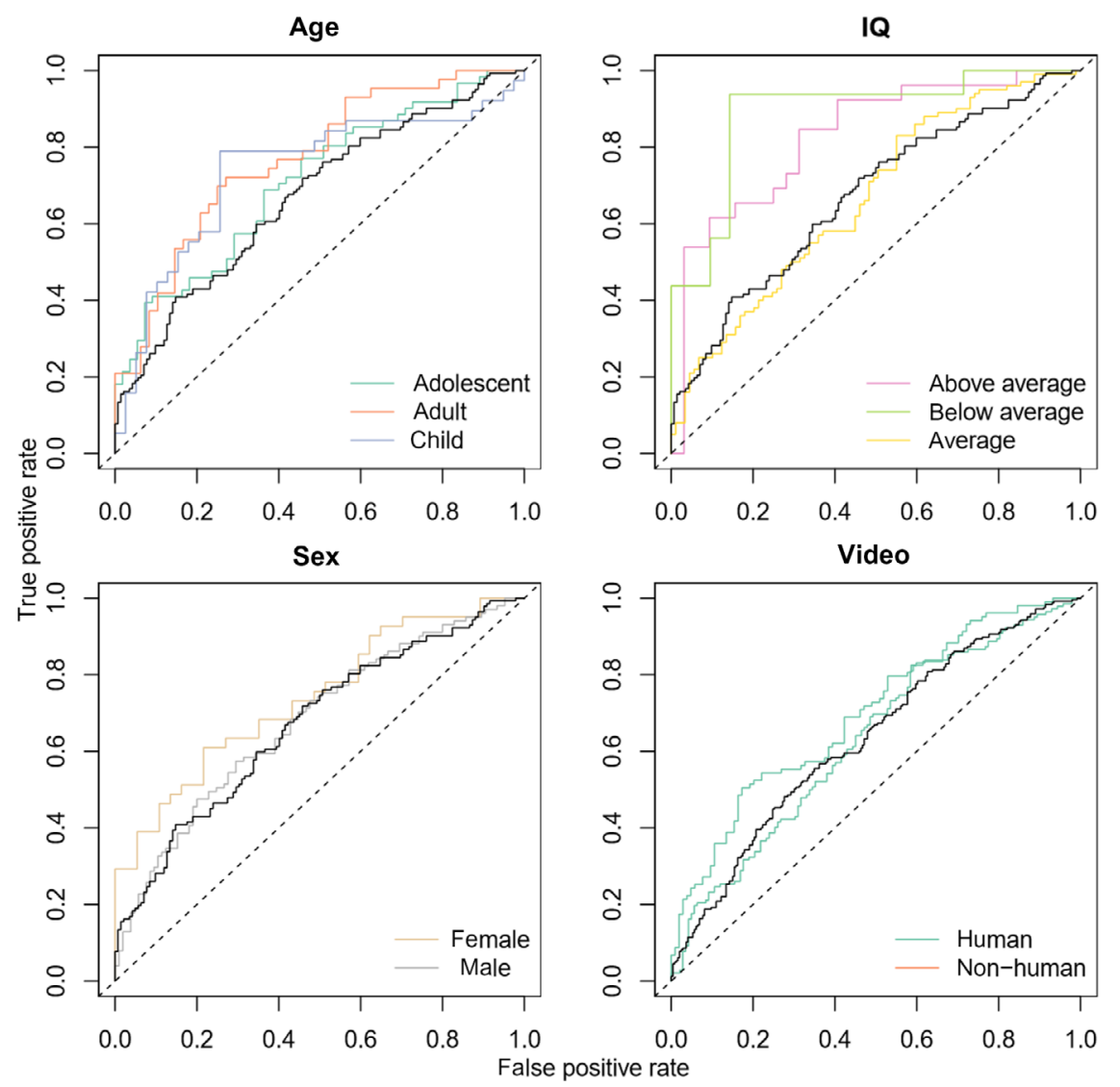

Figure 3 Receiver operating characteristic (ROC) curves of oculomotor function. Black lines represent the ROC in the overall sample. Colored lines represent ROC curves in subgroups or video content conditions. [Colour figure can be viewed at wileyonlinelibrary.com]

Decreased saccade amplitude and duration also correspond to meta-analytic findings of impaired motor coordination in ASD, such as during arm movement, gait, or postural control (Fournier, Hass, Naik, Lodha, \& Cauraugh, 2010). Impaired motor coordination in ASD has been described by increased variation of movement speeds (Gowen \& Hamilton, 2013) and rapid transitions in motor execution ('jerky movements'; Blakemore et al., 2013). This erratic motor execution is reflected in oculomotor function as decreased saccade duration and amplitude.

We propose altered modulation between FON and PBON - that is, pontocerebellar connectivity - as shared underlying neural mechanism of erratic (oculo-)motor execution. Pontocerebellar connectivity modulates finetuning of motor execution and ballistic saccade trajectories (Bodranghien et al., 2016) and has been proposed to explain saccade dysmetria independent of ASD (Choi, Kim, Cho, \& Kim, 2008; Golla et al., 2008). This corresponds well to resting-state findings in our EU-AIMS LEAP sample (Oldehinkel et al., 2018), which reported ASD-specific increased connectivity of the cerebellum with a medial motor network including FON and PBON. In ASD, increased pontocerebellar connectivity has been associated with saccade dysmetria during restricted viewing (Schmitt et al., 2014). A recent review outlined brainstem alterations and its cascading effects on cortical and cerebellar 
development as promising etiological factor in ASD (Dadalko \& Travers, 2018). Thus, altered pontocerebellar motor modulation underlying saccade dysmetria - and other motor execution findings - might be a promising ASD biomarker.

Oculomotor function as feature composite measure was able to determine group affiliation in our heterogenous sample in $67 \%$ of the cases $(\mathrm{AUC}=.670, \mathrm{CI}[0.608,0.733])$. This performance is comparable to the widely established 'social attention' as ASD classifier, which was investigated in toddlers $(\mathrm{AUC}=.710, \mathrm{CI}[0.660,0.760]$; Pierce et al., 2016), younger children $(\mathrm{AUC}=.853$, CI [0.705, 0.964]; Frazier et al., 2016), and older children (AUC $=.721$, CI [0.589, 0.853]; Chevallier et al., 2015). However, these studies assessed more homogenous samples with restricted age and IQ ranges compared to our study. Accordingly, classifier performance was significantly increased (AUC > .733) in more homogenous age, IQ, and sex subgroups (see Table S6), which emphasized those covariates as ASD stratification markers (Charman et al., 2017).

Within the ASD sample, restricted and repetitive behavior (RRB) as measured by ADI-R was positively correlated $(r=.26)$ with velocity main sequence, which describes peak saccade velocity divided by saccade amplitude (see Table S9). This relates saccade dysmetria to the ASD core symptom domain $B$ (i.e., RRB). In addition, decreased atypical sensory responses as measured by a higher SSP score was negatively correlated $(r=-.32)$ with variation in velocity main sequence. Thus, higher sensory symptoms introduce additional variation to saccade behavior within ASD. Sensory symptoms were closely associated with other RRB (Mandy, Charman, \& Skuse, 2012), and RRB was related to ASD-specific gaze patterns (Manyakov et al., 2018). Thus, saccade dysmetria is further concluded as oculomotor indicator of RRB in ASD.

Our findings are preliminary and might not generalize to people with subclinical ASD symptoms, as we applied ADOS clinical cutoff as inclusion criterion. Independent replication is recommended with eye trackers of improved precision and higher sampling rate. Future studies would need to evaluate the specificity of ASD-specific oculomotor function in comparison with ADHD, which is likewise associated with aberrant visual exploration. Future studies may further relate ASD-specific oculomotor function to concurrent gaze behavior including social attention.

\section{Conclusions}

Oculomotor function during visual exploration in ASD is characterized by decreased saccade duration and amplitude. This extends previous findings of inaccurate saccades during restricted viewing to naturalistic visual exploration. This saccade dysmetria is concluded as ASD oculomotor phenotype, which bounds saccades to spatially clustered fixations that attenuate visual exploration independent of human content. Saccade dysmetria was further related to RRB in ASD. Altered pontocerebellar motor modulation is proposed as underlying mechanism of erratic (oculo-)motor execution that contributes to attenuated visual exploration.

\section{Supporting information}

Additional supporting information may be found online in the Supporting Information section at the end of the article:

Table S1. Site ethics.

Table S2. Stimuli characterization.

Table S3. Preprocessing parameters.

Table S4. Linear mixed models overview: oculomotor features $-1 / 3$.

Table S5. Linear mixed models overview: oculomotor features $-2 / 3$.

Table S6. Linear mixed models overview: oculomotor features $-3 / 3$.

Table s7. Age effects.

Table s8. Video content effects.

Table s9. Descriptive correlations with clinical measures in ASD.

\section{Acknowledgements}

This study was funded by EU AIMS and AIMS-2TRIALS. EU-AIMS received support from the IMI Joint Undertaking (JU) under grant agreement no.115300, resources of which are composed of financial contribution from the European Union's Seventh Framework Programme (FP7/2007-2013), from the European Federation of Pharmaceutical Industries and Associations (EFPIA) companies' in kind contribution, and from Autism Speaks. AIMS-2-TRIALS received funding from the IMI 2 JU under grant agreement no. 777394 . The JU receives support from the European Union's Horizon 2020 research and innovation programme and EFPIA, Autism Speaks, Autistica, and the Simons Foundation Autism Research Initiative. The authors acknowledge the contributions of all members of the EU-AIMS LEAP group: Jumana Ahmad, Sara Ambrosino, Bonnie Auyeung, Simon Baron-Cohen, Sarah Baumeister, Christian F. Beckmann, Sven Bölte, Thomas Bourgeron, Carsten Bours, Michael Brammer, Daniel Brandeis, Claudia Brogna, Yvette de Bruijn, Jan K. Buitelaar, Bhismadev Chakrabarti, Tony Charman, Ineke Cornelissen, Daisy Crawley, Flavio Dell'Acqua, Guillaume Dumas, Sarah Durston, Christine Ecker, Jessica Faulkner, Vincent Frouin, Pilar Garcés, David Goyard, Lindsay Ham, Hannah Hayward, Joerg Hipp, Rosemary Holt, Emily J.H. Jones, Prantik Kundu, Meng-Chuan Lai, Xavier Liogier D'ardhuy, Michael V. Lombardo, Eva Loth, David J. Lythgoe, René Mandl, Andre Marquand, Maarten Mennes, Andreas Meyer-Lindenberg, Carolin Moessnang, Declan G.M. Murphy, Bethany Oakley, Laurence O’Dwyer, Marianne Oldehinkel, Bob Oranje, Gahan Pandina, Antonio M. Persico, Barbara Ruggeri, Amber Ruigrok, Jessica Sabet, Roberto Sacco, Antonia 
San José Cáceres, Emily Simonoff, Will Spooren, Julian Tillmann, Roberto Toro, Heike Tost, Jack Waldman, Steve C.R. Williams, Caroline Wooldridge, and Marcel P. Zwiers.

C.M.F. currently receives research funding by the German Ministry of Science and Education (BMBF), the German Research Association (DFG), and the European Commission. She receives royalties for books on ASD, ADHD, and MDD. She has served as consultant for Desitin and Roche in 2017. L.P. received conference support or speaker's fee by Lilly, Shire, and Medice. T.B. served in an advisory or consultancy role for Lundbeck, Medice, Neurim Pharmaceuticals, Oberberg $\mathrm{GmbH}$, and Shire. He received conference support or speaker's fees by Lilly, Medice, Novartis, and Shire. He has been involved in clinical trials conducted by Shire and Viforpharma. He received royalties from Hogrefe, Kohlhammer, CIP Medien, and Oxford University Press. The present work is unrelated to the above grants and relationships. The remaining authors have declared that they have no competing or potential conflicts of interest.

\section{Correspondence}

Nico Bast, Department of Child and Adolescent Psychiatry, Psychosomatics and Psychotherapy, University Hospital, Goethe University Frankfurt am Main, Deutschordenstraße 50, 60528 Frankfurt, Germany; Email: nico.bast@kgu.de

\section{Key points}

- During restricted viewing, previous research indicated saccade dysmetria in autism.

- During visual exploration, we specify saccade dysmetria in autism to decreased saccade duration and amplitude, which suggests attenuated visual exploration.

- Altered pontocerebellar motor modulation is outlined as underlying mechanism that should be further investigated as ASD biomarker.

\section{References}

Andersson, R., Nyström, M., \& Holmqvist, K. (2010). Sampling frequency and eye-tracking measures: how speed affects durations, latencies, and more. Journal of Eye Movement Research, 3, 1-12.

Auyeung, B., Lombardo, M., Heinrichs, M., Chakrabarti, B., Sule, A., Deakin, J., .. \& \& Baron-Cohen, S. (2015). Oxytocin increases eye contact during a real-time, naturalistic social interaction in males with and without autism. Translational Psychiatry, 5, e507.

Bahill, A.T., Clark, M.R., \& Stark, L. (1975). The main sequence, a tool for studying human eye movements. Mathematical Biosciences, 24, 191-204.

Bates, D., Maechler, M., Bolker, B., \& Walker, S. (2014). lme4: Linear mixed-effects models using Eigen and S4. R package version, 1(7), 1-23.

Bedford, R., Gliga, T., Shephard, E., Elsabbagh, M., Pickles, A., Charman, T. \& Johnson, M.H. (2017). Neurocognitive and observational markers: prediction of autism spectrum disorder from infancy to mid-childhood. Molecular Autism, 8, 49.

Blakemore, S.-J., Cook, J.L., \& Press, C. (2013). Atypical basic movement kinematics in autism spectrum conditions. Brain, 136, 2816-2824.

Bodranghien, F., Bastian, A., Casali, C., Hallett, M., Louis, E.D., Manto, M., .. \& \& van Dun, K. (2016). Consensus paper: Revisiting the symptoms and signs of cerebellar syndrome. The Cerebellum, 15, 369-391.

Bottini, S. (2018). Social reward processing in individuals with autism spectrum disorder: A systematic review of the social motivation hypothesis. Research in Autism Spectrum Disorders, 45, 9-26.

Charman, T., Loth, E., Tillmann, J., Crawley, D., Wooldridge, C., Goyard, D. ... \& Buitelaar, J.K. (2017). The EU-AIMS Longitudinal European Autism Project (LEAP): Clinical characterisation. Molecular Autism, 8, 27.

Chevallier, C., Kohls, G., Troiani, V., Brodkin, E.S., \& Schultz, R.T. (2012). The social motivation theory of autism. Trends in Cognitive Sciences, 16, 231-239.
Chevallier, C., Parish-Morris, J., McVey, A., Rump, K.M., Sasson, N.J., Herrington, J.D. \& Schultz, R.T. (2015). Measuring social attention and motivation in autism spectrum disorder using eye-tracking: Stimulus type matters. Autism Research, 8, 620-628.

Chica, A.B., Bartolomeo, P., \& Lupiáñez, J. (2013). Two cognitive and neural systems for endogenous and exogenous spatial attention. Behavioural Brain Research, 237, 107123.

Choi, K.-D., Kim, H.-J., Cho, B.M., \& Kim, J.S. (2008). Saccadic adaptation in lateral medullary and cerebellar infarction. Experimental Brain Research, 188, 475.

Corbetta, M. (1998). Frontoparietal cortical networks for directing attention and the eye to visual locations: Identical, independent, or overlapping neural systems? Proceedings of the National Academy of Sciences of the United States of America, 95, 831-838.

Corbetta, M., Akbudak, E., Conturo, T.E., Snyder, A.Z., Ollinger, J.M., Drury, H.A., .. \& \& Shulman, G.L. (1998). A common network of functional areas for attention and eye movements. Neuron, 21, 761-773.

Corbetta, M., Patel, G., \& Shulman, G.L. (2008). The reorienting system of the human brain: From environment to theory of mind. Neuron, 58, 306-324.

Dadalko, O.I., \& Travers, B.G. (2018). Evidence for brainstem contributions to autism spectrum disorders. [Review]. Frontiers in Integrative Neuroscience, 12, 47.

de Urabain, I.R.S., Johnson, M.H., \& Smith, T.J. (2014). GraFIX: A semiautomatic approach for parsing low-and high-quality eye-tracking data. Behavior Research Methods, 47, 53-72.

Fournier, K.A., Hass, C.J., Naik, S.K., Lodha, N., \& Cauraugh, J.H. (2010). Motor coordination in autism spectrum disorders: A synthesis and meta-analysis. Journal of Autism and Developmental Disorders, 40, 1227-1240.

Frazier, T.W., Klingemier, E.W., Beukemann, M., Speer, L., Markowitz, L., Parikh, S., ... \& Strauss, M.S. (2016). Development of an objective autism risk index using remote 
eye tracking. Journal of the American Academy of Child \& Adolescent Psychiatry, 55, 301-309.

Frazier, T.W., Strauss, M., Klingemier, E.W., Zetzer, E.E., Hardan, A.Y., Eng, C., \& Youngstrom, E.A. (2017). A metaanalysis of gaze differences to social and nonsocial information between individuals with and without autism. Journal of the American Academy of Child \& Adolescent Psychiatry, 56, 546-555.

Gliga, T., Bedford, R., Charman, T., Johnson, M.H., BaronCohen, S., Bolton, P., ... \& Tucker, L. (2015). Enhanced visual search in infancy predicts emerging autism symptoms. Current Biology, 25, 1727-1730.

Gliga, T., Smith, T.J., Likely, N., Charman, T., \& Johnson, M.H. (2015). Early visual foraging in relationship to familial risk for autism and hyperactivity/inattention. Journal of attention disorders, 22, 839-847.

Golla, H., Tziridis, K., Haarmeier, T., Catz, N., Barash, S., \& Thier, P. (2008). Reduced saccadic resilience and impaired saccadic adaptation due to cerebellar disease. European Journal of Neuroscience, 27, 132-144.

Gotham, K., Pickles, A., \& Lord, C. (2009). Standardizing ADOS scores for a measure of severity in autism spectrum disorders. Journal of autism and developmental disorders, 39, 693-705.

Gowen, E., \& Hamilton, A. (2013). Motor abilities in autism: A review using a computational context. Journal of Autism and Developmental Disorders, 43, 323-344.

Green, P., \& MacLeod, C.J. (2016). SIMR: An R package for power analysis of generalized linear mixed models by simulation. Methods in Ecology and Evolution, 7, 493-498.

Guerrasio, L., Quinet, J., Büttner, U., \& Goffart, L. (2010). Fastigial oculomotor region and the control of foveation during fixation. Journal of Neurophysiology, 103, 19882001.

Ho, D., Imai, K., King, G., Stuart, E., \& Whitworth, A. (2018). Package 'MatchIt': Version 3.0.2.

Itti, L., \& Koch, C. (2001). Computational modelling of visual attention. Nature Reviews Neuroscience, 2, 194-203.

Johnson, B.P., Lum, J.A.G., Rinehart, N.J., \& Fielding, J. (2016). Ocular motor disturbances in autism spectrum disorders: Systematic review and comprehensive metaanalysis. Neuroscience \& Biobehavioral Reviews, 69, 260279.

Johnson, B.P., Rinehart, N., Papadopoulos, N., Tonge, B., Millist, L., White, O. \& Fielding, J. (2012). A closer look at visually guided saccades in autism and Asperger's disorder. Frontiers in Integrative Neuroscience, 6, 99.

Jones, E.J., Venema, K., Earl, R., Lowy, R., Barnes, K., Estes, A., Webb, S.J. (2016). Reduced engagement with social stimuli in 6-month-old infants with later autism spectrum disorder: A longitudinal prospective study of infants at high familial risk. Journal of Neurodevelopmental Disorders, 8, 7.

Kessler, R.C., Adler, L., Ames, M., Demler, O., Faraone, S., Hiripi, E., ... Walters, E.E. (2005). The World Health Organization Adult ADHD Self-Report Scale (ASRS): A short screening scale for use in the general population. Psychological Medicine, 35, 245-256.

Kuznetsova, A., Brockhoff, P.B., \& Christensen, R.H.B. (2017). lmerTest package: Tests in linear mixed effects models. Journal of Statistical Software, 82, 13.

Landry, O., \& Parker, A. (2013). A meta-analysis of visual orienting in autism. Frontiers in Human Neuroscience, 7, 833.

Loth, E., Charman, T., Mason, L., Tillmann, J., Jones, E.J.H., Wooldridge, C., .. \& \& Buitelaar, J.K. (2017). The EU-AIMS Longitudinal European Autism Project (LEAP): Design and methodologies to identify and validate stratification biomarkers for autism spectrum disorders. Molecular Autism, 8, 24 .

Mandy, W.P., Charman, T., \& Skuse, D.H. (2012). Testing the construct validity of proposed criteria for DSM-5 autism spectrum disorder. Journal of the American Academy of Child \& Adolescent Psychiatry, 51, 41-50.

Manyakov, N.V., Bangerter, A., Chatterjee, M., Mason, L., Ness, S., Lewin, D., ... \& Pandina, G. (2018). Visual exploration in autism spectrum disorder: Exploring age differences and dynamic features using recurrence quantification analysis. Autism Research, 11, 1554-1566.

Mill, R.D., O'Connor, A.R., \& Dobbins, I.G. (2016). Pupil dilation during recognition memory: Isolating unexpected recognition from judgment uncertainty. Cognition, 154, 8194.

Mosconi, M.W., Luna, B., Kay-Stacey, M., Nowinski, C.V., Rubin, L.H., Scudder, C., ... \& Sweeney, J.A. (2013). Saccade adaptation abnormalities implicate dysfunction of cerebellar-dependent learning mechanisms in autism spectrum disorders (ASD). PLoS ONE, 8, e63709.

Munoz, D.P., \& Everling, S. (2004). Look away: The antisaccade task and the voluntary control of eye movement. Nature Reviews Neuroscience, 5, 218.

Nakamagoe, K., Iwamoto, Y., \& Yoshida, K. (2000). Evidence for brainstem structures participating in oculomotor integration. Science, 288, 857-859.

Nyström, P., Gliga, T., Nilsson Jobs, E., Gredebäck, G., Charman, T., Johnson, M.H., .. \& Falck-Ytter, T. (2018). Enhanced pupillary light reflex in infancy is associated with autism diagnosis in toddlerhood. Nature Communications, 9, 1678.

Oldehinkel, M., Mennes, M., Marquand, A., Charman, T., Tillmann, J., Ecker, C., .. \& \& EU-AIMS LEAP Group (2018). Altered connectivity between cerebellum, visual, and sensory-motor networks in autism spectrum disorder: Results from the EU-AIMS Longitudinal European Autism Project. Biological Psychiatry: Cognitive Neuroscience and Neuroimaging, 4, 260-270.

Pierce, K., Marinero, S., Hazin, R., McKenna, B., Barnes, C.C., \& Malige, A. (2016). Eye tracking reveals abnormal visual preference for geometric images as an early biomarker of an autism spectrum disorder subtype associated with increased symptom severity. Biological Psychiatry, 79, 657-666.

$\mathrm{R}$ Core Team (2014). R: A language and environment for statistical computing (vol. 3). Vienna, Austria: R Foundation for Statistical Computing.

Radach, R., Deubel, H., Vorstius, C., \& Hofmann, M. (2017). Abstracts of the 19th European Conference on Eye Movements 2017. Journal of Eye Movement Research, 10, 6.

Renner, P., Grofer Klinger, L., \& Klinger, M.R. (2006). Exogenous and endogenous attention orienting in autism spectrum disorders. Child Neuropsychology, 12, 361-382.

Robin, X., Turck, N., Hainard, A., Tiberti, N., Lisacek, F., Sanchez, J.C., \& Müller, M. (2011). pROC: An open-source package for $\mathrm{R}$ and $\mathrm{S}+$ to analyze and compare ROC curves. BMC Bioinformatics, $12,77$.

Russell, L. (2018). Emmeans: Estimated Marginal Means, aka Least-Squares Means. R package version 1.2.

Schmitt, L.M., Cook, E.H., Sweeney, J.A., \& Mosconi, M.W. (2014). Saccadic eye movement abnormalities in autism spectrum disorder indicate dysfunctions in cerebellum and brainstem. Molecular Autism, 5, 47.

Simonoff, E., Pickles, A., Charman, T., Chandler, S., Loucas, T., \& Baird, G. (2008). Psychiatric disorders in children with autism spectrum disorders: prevalence, comorbidity, and associated factors in a population-derived sample. Journal of the American Academy of Child \& Adolescent Psychiatry, 47, 921-929.

Sun, X., \& Xu, W. (2014). Fast implementation of DeLong's algorithm for comparing the areas under correlated receiver operating characteristic curves. IEEE Signal Processing Letters, 21, 1389-1393.

Takarae, Y., Luna, B., Minshew, N.J., \& Sweeney, J.A. (2008). Patterns of visual sensory and sensorimotor abnormalities 
in autism vary in relation to history of early language delay. Journal of the International Neuropsychological Society, 14, 980-989.

Takarae, Y., Minshew, N.J., Luna, B., Krisky, C.M., \& Sweeney, J.A. (2004). Pursuit eye movement deficits in autism. Brain, 127, 2584-2594.

Takarae, Y., Minshew, N., Luna, B., \& Sweeney, J. (2004). Oculomotor abnormalities parallel cerebellar histopathology in autism. Journal of Neurology, Neurosurgery \& Psychiatry, 75, 1359-1361.

Wang, C.-A., \& Munoz, D.P. (2015). A circuit for pupil orienting responses: Implications for cognitive modulation of pupil size. Current Opinion in Neurobiology, 33, 134-140.
Wang, S., Jiang, M., Duchesne, X.M., Laugeson, E.A., Kennedy, D.P., Adolphs, R., \& Zhao, Q. (2015). Atypical visual saliency in autism spectrum disorder quantified through model-based eye tracking. Neuron, 88, 604-616.

Wass, S.V., Jones, E.J., Gliga, T., Smith, T.J., Charman, T., \& Johnson, M.H. (2015). Shorter spontaneous fixation durations in infants with later emerging autism. Scientific Reports, 5, 8284.

Wass, S.V., \& Smith, T.J. (2014). Individual differences in infant oculomotor behavior during the viewing of complex naturalistic scenes. Infancy, 19, 352-384.

Accepted for publication: 23 April 2020 